\title{
IRST Infrared Background analysis of Bay environments
}

\author{
Piet B.W. Schwering ${ }^{1}$, Dirk F. Bezuidenhout ${ }^{2}$, Willem H. Gunter ${ }^{3}$, Francois P.J. le Roux ${ }^{2}$, \\ Rheinhardt H. Sieberhagen ${ }^{2}$ \\ ${ }^{1}$ TNO Defence, Security and Safety, P.O. Box 96864, NL-2509 JG The Hague, Netherlands, piet.schwering @tno.nl \\ ${ }^{2}$ CSIR DPSS, P.O. Box 395, Pretoria, 0001, South Africa, dbezuide@ csir.co.za \\ ${ }^{3}$ Institute for Maritime Technology (IMT), Martello Road, Simon's Town 7995, South Africa, whg@imt.co.za
}

\begin{abstract}
Present-day naval operations take place in coastal environments as well as narrow straits all over the world. Coastal environments around the world are exhibiting a number of threats to naval forces. In particular a large number of asymmetric threats can be present in environments with cluttered backgrounds as well as rapidly varying atmospheric conditions. During trials executed in False Bay a large amount of target, background and atmosphere data was gathered that is of use in analysis of optical characteristics of targets and backgrounds. During the trials a variety of backgrounds were recorded. We have used these backgrounds to validate the TNO background model MIBS to incorporate also coastal backgrounds and sunlit sea backgrounds. In the paper we show results of the background analysis, for coastal bay backgrounds. In particular the detection of small targets by automatic system may be hampered by small surface structure variations at the surface and near the horizon. The data that we analyzed are sea surface structure, temporal behaviour, and spectral differences during different environmental conditions that occurred during the trials. This data is essential to feed detection algorithms, and performance models for the assessment of sensor performance in coastal environment. Some sensor management approaches for application in IRST systems is discussed.
\end{abstract}

Keywords: Infrared backgrounds, coastal bay analysis, small surface target contrasts, IRST application.

\section{INTRODUCTION}

More and more, coastal environments are the terrain of operation of naval forces. All over the world, operation in narrow straits and bay environments pose the threats of presence of small unknown surface vessels. In particular, large numbers of asymmetric threats can be present in environments with highly cluttered backgrounds as well as rapidly varying atmospheric conditions. Threat contrasts may be low and varying in littoral environments, and the amount of background clutter can be severe. Electro-optical sensors, used for detection and classification, should be optimized in order to increase reaction time. The assessment of classification task is important for this procedure.

Present-day electro-optical sensor suites are used for diverse tasks as detection, classification and identification. These inherently passive systems, such as infrared and visible light sensors, can definitely improve situational awareness. For application over long range elevated sensor masts are best suited for the surveillance task and improvement of the operational picture. Effective combination of electro-optical sensor technology and advanced signal processing will lead to new system concepts. Of greatest importance is the analysis of threat information from the high spatial-resolution imagery recorded with different sensors. As electro-optical sensors do not have all-weather capability the performance degradation in adverse scenarios, such as caused by the varying conditions in coastal environments, can be relatively low. The use of adaptive sensor management techniques can support the effective application of electro-optical sensors in these environments. The use of background models and vertical background radiance and clutter profiles can be important to optimize detection sensor systems ${ }^{1,2}$.

In this paper we present the analysis of data recorded on backgrounds in bay environment. The data shows clear variations in background radiance data and clutter during different conditions. This shows the variation that can be present in the environment. In Section 2 we present a very short description of the trials. Section 3 shows results on the variability of infrared background. Section 4 gives results of background simulations for these scenarios, and gives a

Infrared Technology and Applications XXXIV, edited by Bjørn F. Andresen, Gabor F. Fulop, Paul R. Norton, Proc. of SPIE Vol. 6940, 69401E, (2008) · 0277-786X/08/\$18 · doi: 10.1117/12.778449 
discussion of the validity of the models. Section 5 gives results on the variability of infrared radiation of small surface targets and their interaction with the sea in the form of wake structure. In Section 6 we discuss an approach to derivation of sensor management information. The paper finishes with the conclusions.

\section{INFRARED BACKGROUND MEASUREMENTS}

In June 2007 the TNO (The Netherlands), CSIR and IMT (South Africa) performed an experiment into the analysis of optical characteristics of small surface targets. For this experiment False Bay (South Africa) was chosen as the measurement location. A full description of the experiment is given in Schwering et al. (2007) ${ }^{3}$. We refer to that paper for information on sensors, experiments and environments used in the trials. Beside optical target characteristics, valuable recordings were taken of backgrounds, sea, sky and land, as well as of atmospheric parameters, such as scintillation and blur. A number of targets were used in the trials, such as a small scientific work boat and a small harbour patrol boat. These will be visible in the figures hereafter. The meteorological conditions during the two week trials can best be described as varying winter conditions. These were ranging from sunny warm days up to cold, rainy and stormy conditions. These conditions gave a variety of sea state conditions in the bay. We chose to perform separate analyses for target, atmosphere and background. In this paper we describe the background analysis.

\section{VARIABILITY OF INFRARED BACKGROUNDS}

In this section we analyse a number of different infrared backgrounds recorded during the False Bay trials. Examples of backgrounds (and targets) that were measured in the thermal MWIR and LWIR, using radiometric sensor systems, are shown in Figure 1. For these figures (numbered a-e) the dates and times as well as meteorological conditions are given in Table 1. These data was recorded with a CEDIP Jade MWIR camera and an Agema THV1000 CLR LWIR camera ${ }^{3}$.

Table 1. Environmental Conditions

\begin{tabular}{|c|c|c|c|c|c|c|c|c|c|c|c|c|}
\hline Serial & Date & Time & Wind & VIS & Solar & $\mathrm{CC}$ & Rel Sun Az & Sun El & Tair & Tsea & $\mathbf{R H}$ & $p$ \\
\hline & & & $\mathrm{m} / \mathrm{s}$ & km & $\mathrm{W} / \mathrm{m}^{2}$ & $x / 8$ & deg & deg & deg & deg & $\%$ & $\mathrm{hPa}$ \\
\hline a) & 6-Jun & 19:09 & 2.9 & 40 & 0 & $8 / 8$ & 66 & -17 & 14 & 13.4 & 82 & 1008 \\
\hline b) & 7-Jun & $10: 04$ & 5.3 & 25 & 291 & $4 / 8$ & 48 & 22 & 15.9 & 13.4 & 74 & 1008 \\
\hline c) & 12-Jun & $10: 06$ & 4.7 & 60 & 282 & $2 / 8$ & 47 & 22 & 14.3 & 13.4 & 62 & 1023 \\
\hline d) & 13-Jun & $10: 04$ & 7.6 & 50 & 341 & $0 / 8$ & 48 & 21 & 13.4 & 13.4 & 62 & 1023 \\
\hline e) & 13-Jun & $12: 07$ & 7.3 & 50 & 575 & $0 / 8$ & 19 & 32 & 13.5 & 13.4 & 59 & 1022 \\
\hline
\end{tabular}

Vertical radiance profiles were calculated by taking the mean of each row of pixels in the image and plotting that against the elevation angle (relative to the horizon, negative for sea background). Vertical profiles of the background variation were also established by calculating the standard deviation in the radiance for each row in the image and plotting this against the elevation angle. The results of the vertical mean and standard deviation radiance profiles appear respectively in Figure 2 and Figure 3. The same numbering scheme a-e is used as in the previous figure.

From the figures we see that there is a large variation in background radiance and clutter profiles for conditions that are in just slightly varying meteorological conditions. Radiance variations of more than $10 \%$ occur while profiles can be crossing, in cases of different air-sea-temperature differences. Land was apparently always warmer than sea, while the MWIR clutter on land is larger, and the LWIR clutter on sea. Horizon and target clearly show up in the clutter graphs.

Both the mean and RMS variation level of the background depend on many factors including the amount of cloud cover, sea state (wind speed), relative sun azimuth angle (azimuth angle separation between observer line-of-sight and sun) and sun elevation angle (time of day). Table 1 contains the important environmental parameters for each of the images shown in the figures 1-3.

Looking at the sea background mean levels in Figure 2 the largest influence is due to the amount of cloud cover and reflected (MWIR) sunlight. Comparing the clutter levels in Figure 3, the sea background variation increases with increasing wind speed (sea state), decreasing relative sun azimuth angle, decreasing cloud cover and increasing sun irradiance (condition a), c), b), d) and e) - in order from lowest to highest noise level). The largest difference between the sea background variation in the MWIR and LWIR infrared band is the relative insensitivity of the LWIR to sunglint clutter (condition e). The problem of sunglint clutter from the sea surface should even be worse for sensors operating at 
shorter wavelengths (e.g. SWIR and VIS bands). LWIR sensors $(8-12 \mu \mathrm{m})$ will therefore experience the lowest false alarm rates due to sunglint from the sea surface during day time.

Changes in the MWIR sea and land background can also be caused by rapid cloud movement. An example is shown in Figure 4, where both the mean and standard deviation background radiance has changed over a 40 second period due to cloud movement and partial sun irradiance on different parts of the sea and land background.
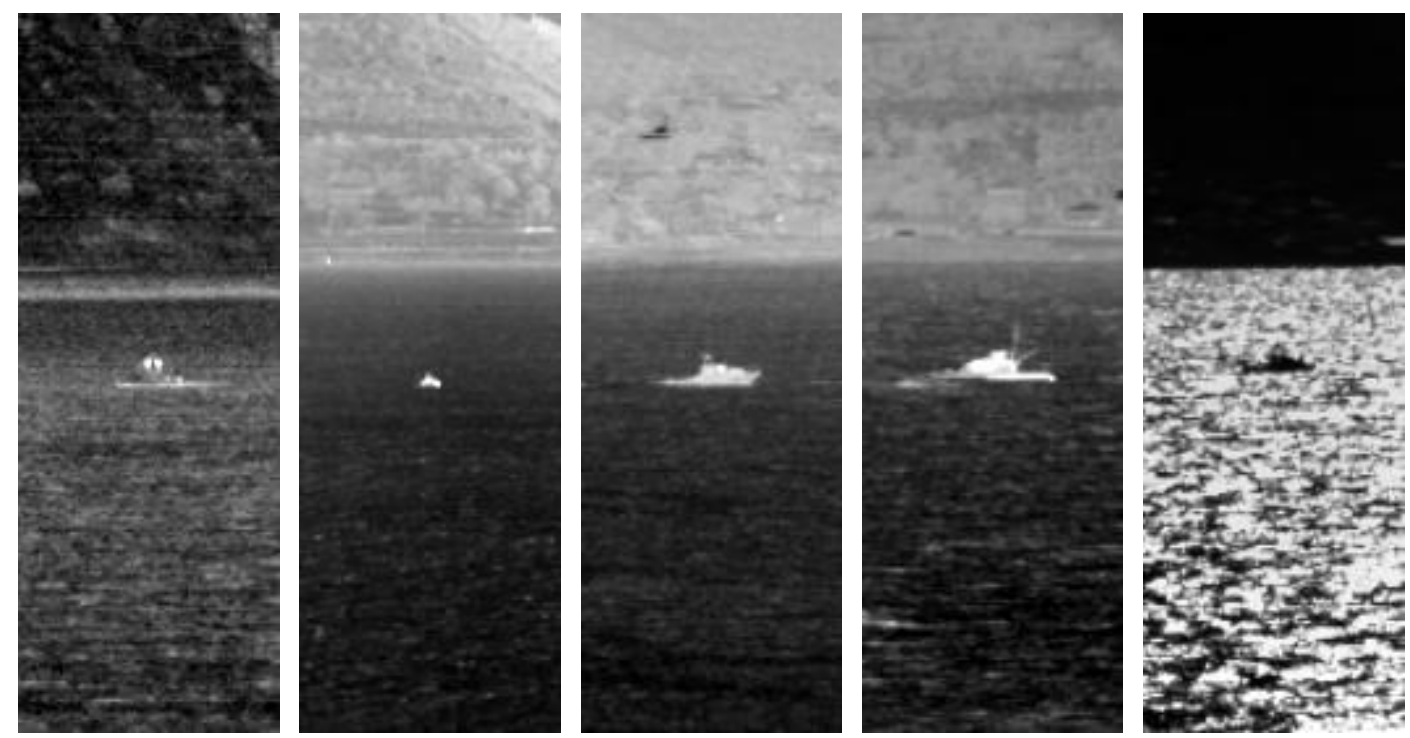

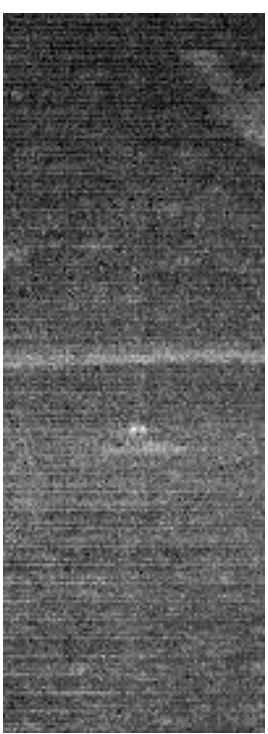

a)

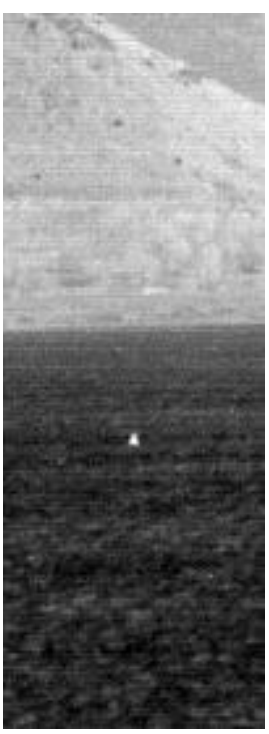

b)

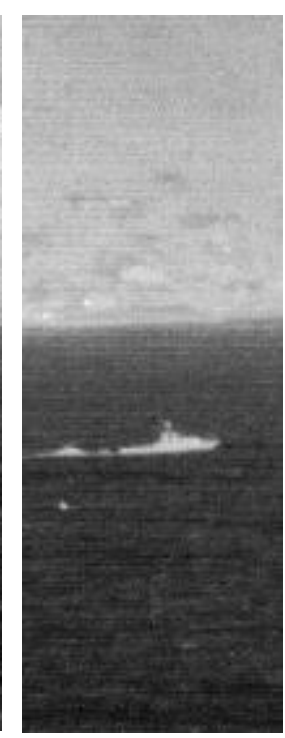

c)

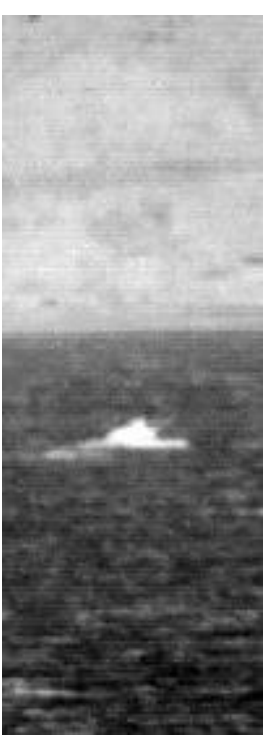

d)

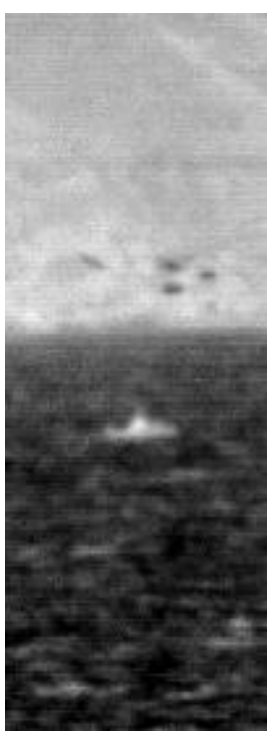

e)

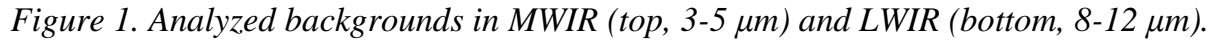



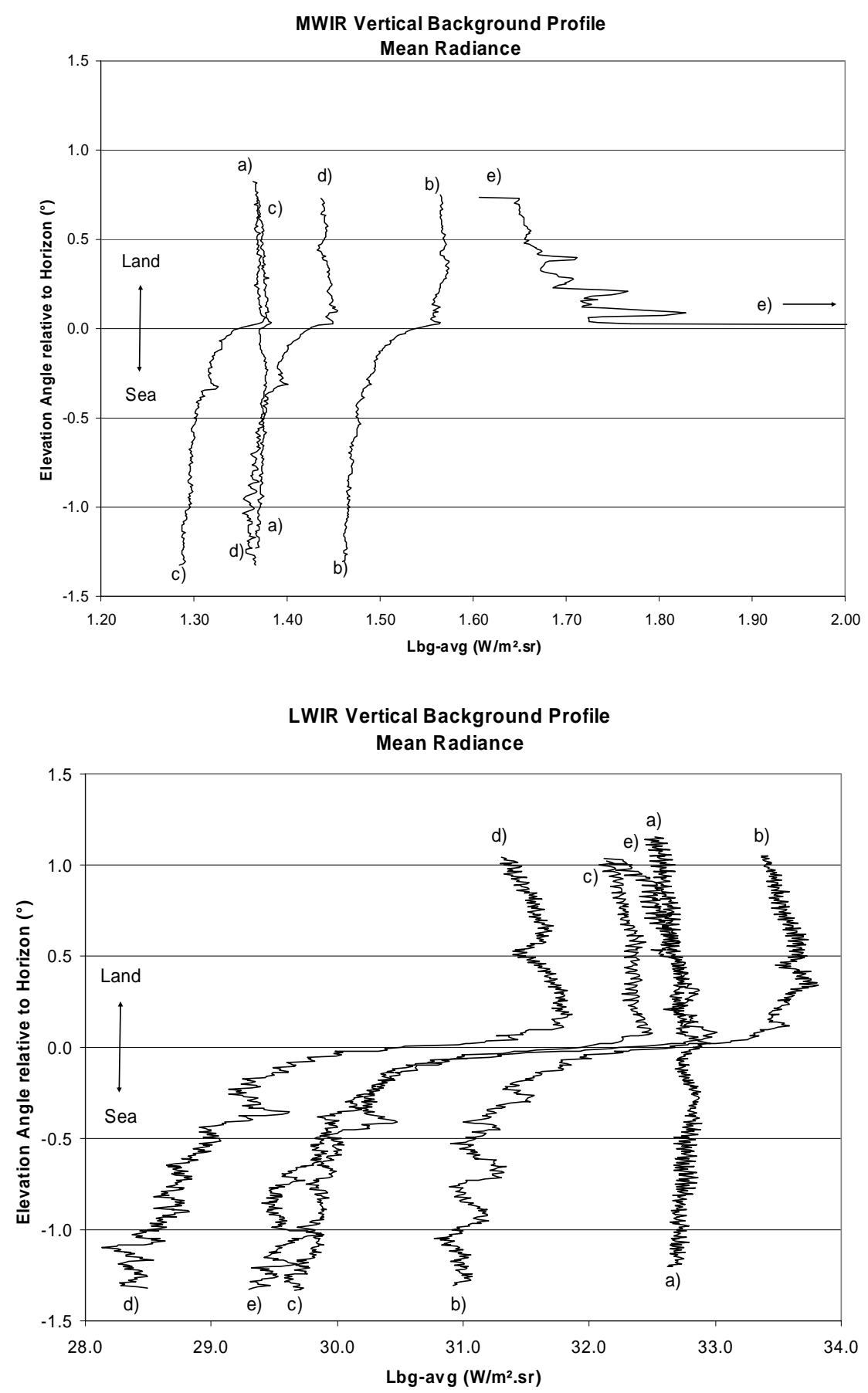

Figure 2. Vertical mean radiance profile in MWIR (top) \& LWIR (bottom). 

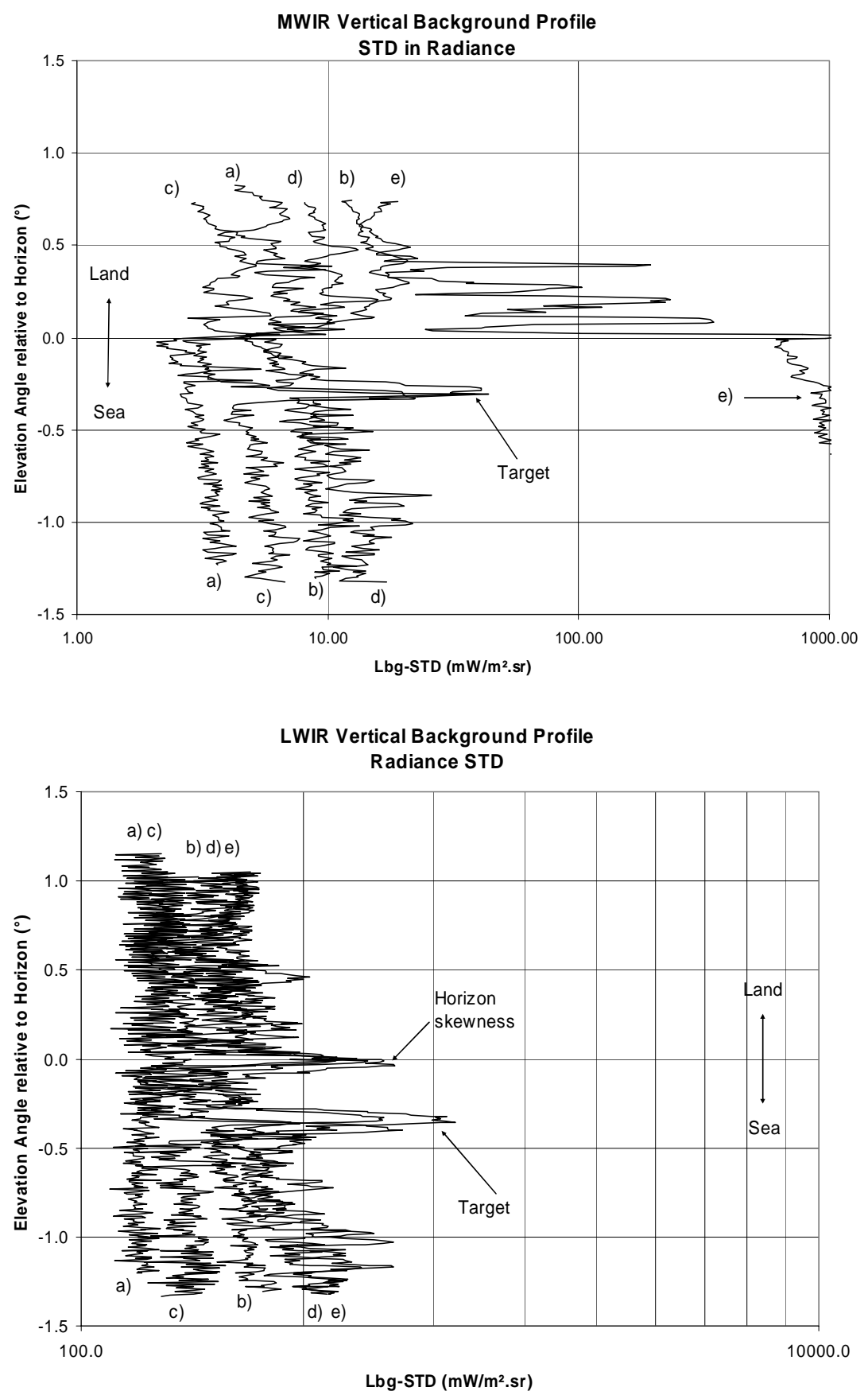

Figure 3. Background clutter (RMS variation) in MWIR (top) and LWIR (bottom). 

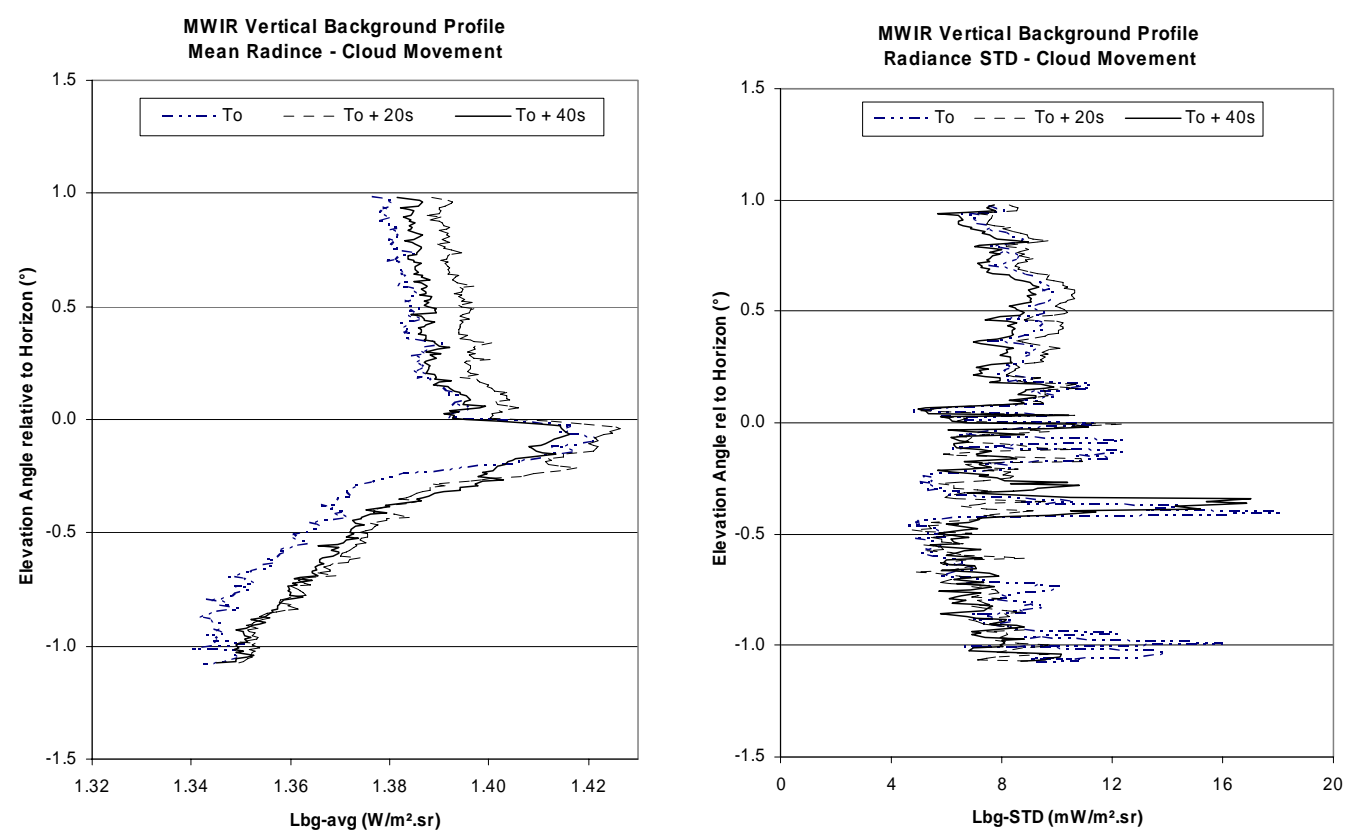

Figure 4. Changes in MWIR background due to cloud movement (average left, standard deviation right).

An example of another source of clutter appears in Figure 5, showing low flying migrating birds. This can cause serious false alarms problems for IRST systems. As can be seen in the figure the standard deviation for the birds can be substantially larger than that of the surface vessel.

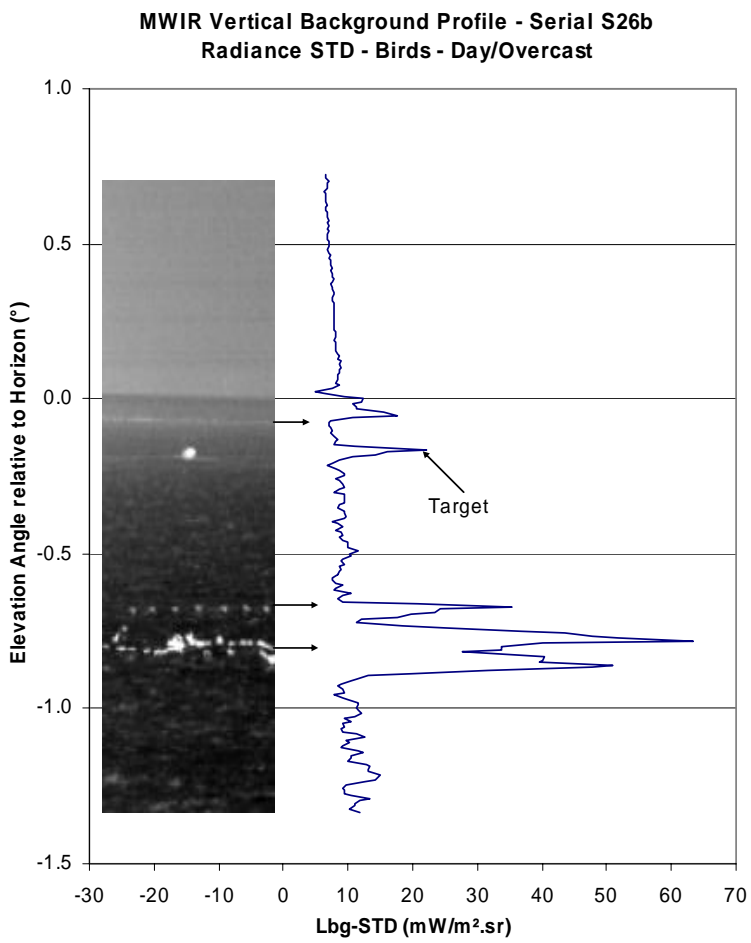

Figure 5. MWIR Clutter from low flying birds. 


\section{INFRARED BACKGROUND SIMULATIONS}

In the last decade TNO has spent ample time to develop a maritime background model for the analysis, development and evaluation of infrared search and track systems. This Maritime Infrared Background Simulator $\left(\mathrm{MIBS}^{4}\right)$ generates images in MWIR and LWIR bands of the maritime horizon background. It was designed to perform well in terms of background radiance and clutter prediction near the optical ocean horizon. The basic information is vertical background radiance and clutter profiles (Schwering (1991) $)^{5}$. Several validation issues were described in previous papers ${ }^{1}$. The latter years interest has moved towards the littoral conditions. Model extensions with spectral information, clouds, and sunglint have been performed. Coastal backgrounds have been implemented, but with the lack of sufficient data complexity, the use of additional infrared data in bay environments gave the opportunity to validate its behaviour in these coastal conditions.

In Figure 6 we present MWIR and LWIR imagery for the same conditions a-e as in the figures 1-3. For these calculations we used the meteorological data given in Table 1. The selection of background profile in the MIBS environment is based on air temperature, sea temperature, relative humidity, wind speed, and solar irradiance. When we compare the simulated images from Figure 6 with the images from recorded Figure 1 we note the same basic structures: horizon, land and sea backgrounds. The sea shows wave structure with white caps on top. Land background radiance levels are dominated by the air temperature, and sea clutter corresponds qualitatively well with the same moments between the two figures. The largest clutter occurs in images d) and e) and the lowest apparently in image b). Later in this section this will be quantified, as the image scaling procedure has an impact on our appreciation of the data.

In Figure 7 we present the LWIR radiance and clutter variation data for the conditions a-e. The land background is at the right (elevations large than zero) and the sea background is at the left of the graphs. In the figure we observe the following characteristics in the profiles.

Land background radiance is well correlated with the recorded information of the previous section. The land background value shows a relation with air temperature. The sea background values in the profile goes down to the value of $30.6 \mathrm{~W} / \mathrm{m}^{2} \mathrm{sr}$, almost identical to the values of all the conditions. This is due to the profile selection process in the simulations, where minor profile corrections occur due to the difference in air temperature. The sea temperature was chosen the same in the simulations of all the conditions.

For the clutter variation, a similar effect occurs in the conditions a-e for the simulated data as for the measured data. From the simulated data clutter increased in the order of case a-c-b-e-d. The order is almost similar to the clutter order of Figure 3 (a-c-b-d-e in LWIR). Data numbers are well in line between the simulated data and the recorded data. The average clutter levels of the simulations are between 0.1 (condition a) and approximately $0.25 \mathrm{~W} / \mathrm{m}^{2} \mathrm{sr}$ (condition d). Peaks up to $0.45 \mathrm{~W} / \mathrm{m}^{2}$ sr occur. From the measured data average clutter levels are between 0.15 (condition a) and 0.34 $\mathrm{W} / \mathrm{m}^{2} \mathrm{sr}$ (condition e). Higher variances in these clutter levels occur at the higher clutter levels with respect to the lower clutter levels. This shows strong peaks occur also the recorded data.

In general we can state that accurate background clutter levels have been simulated in the False Bay conditions. In the future, background radiance levels can be improved in the simulations by making the sea background more dependent on environmental temperatures (sea and air).
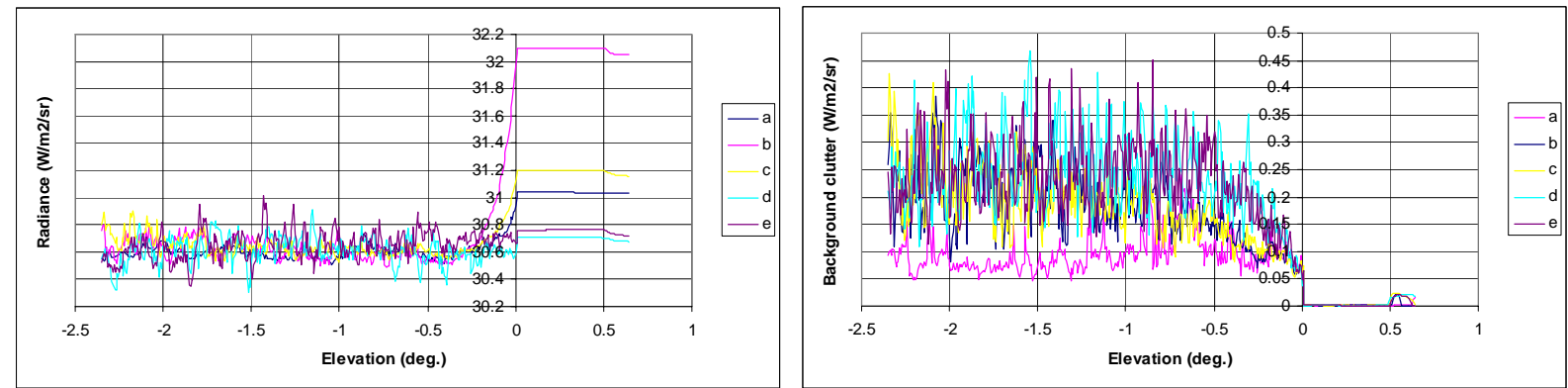

Figure 7. Vertical LWIR radiance (left) and clutter (right) profiles of the MIBS simulations. 

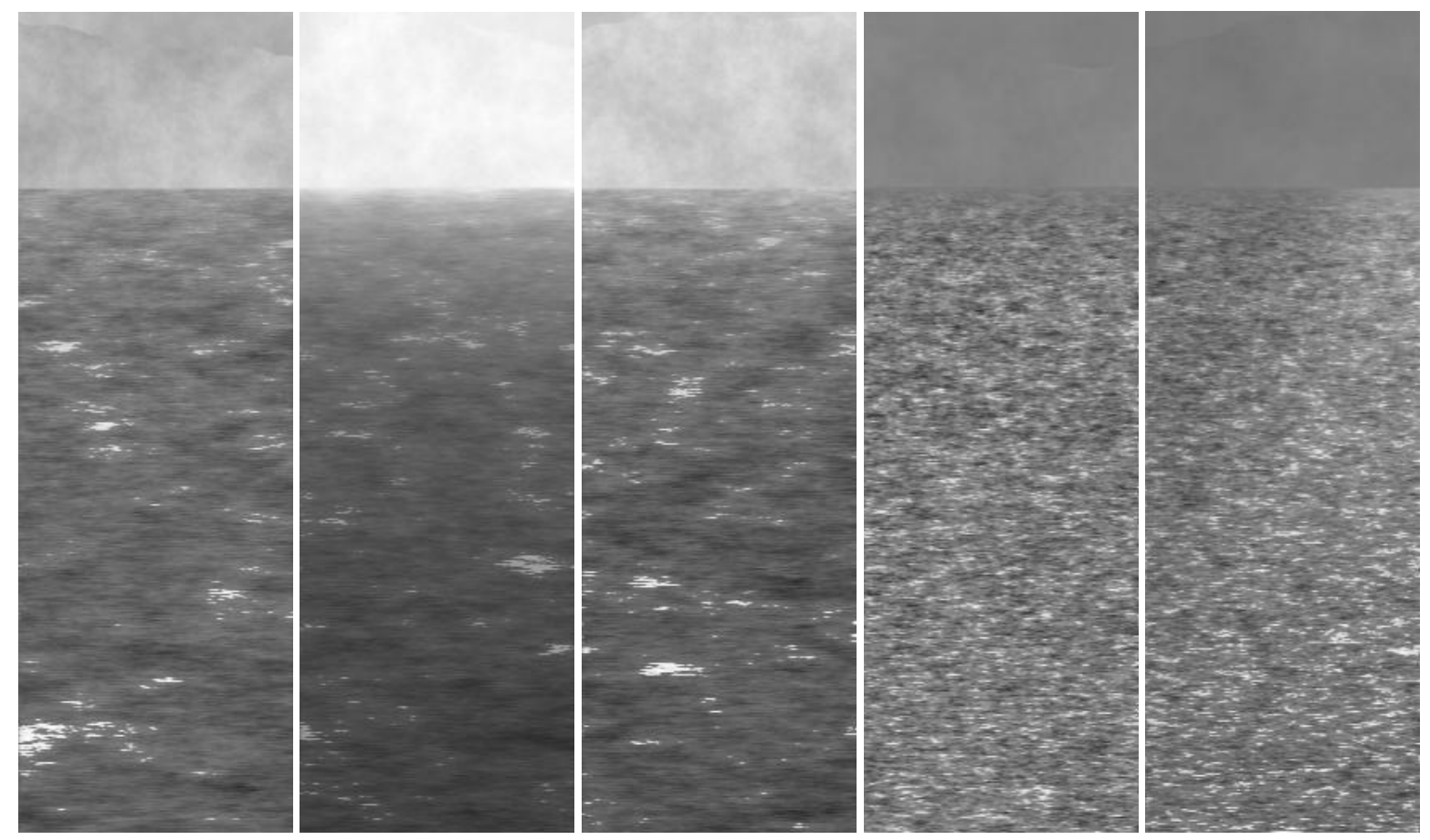

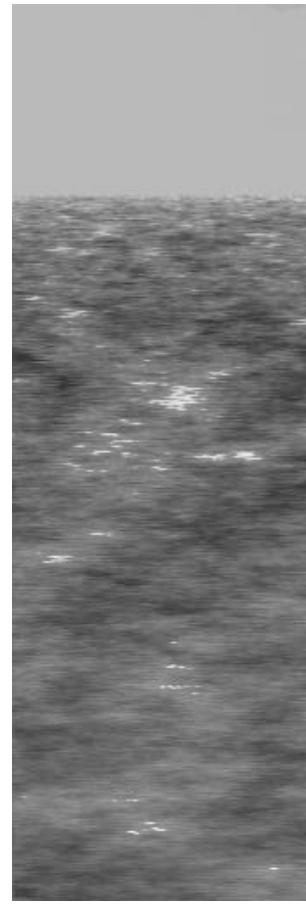

a)

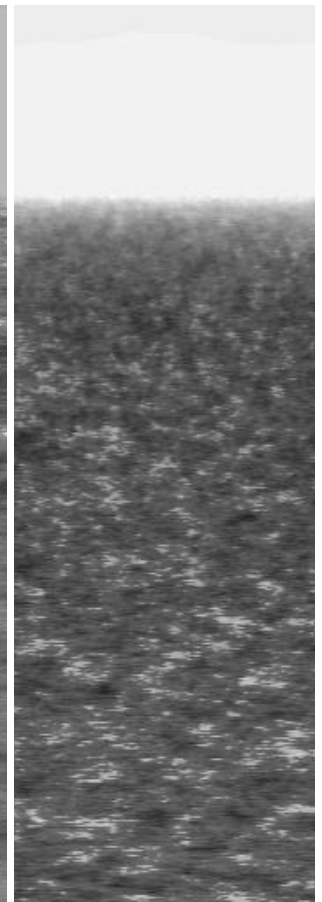

b)

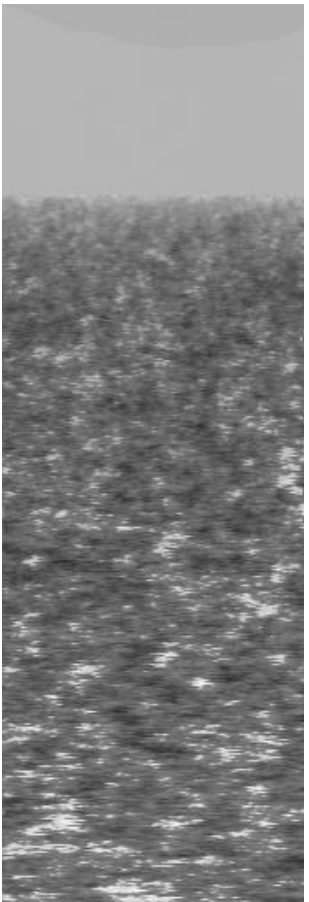

c)

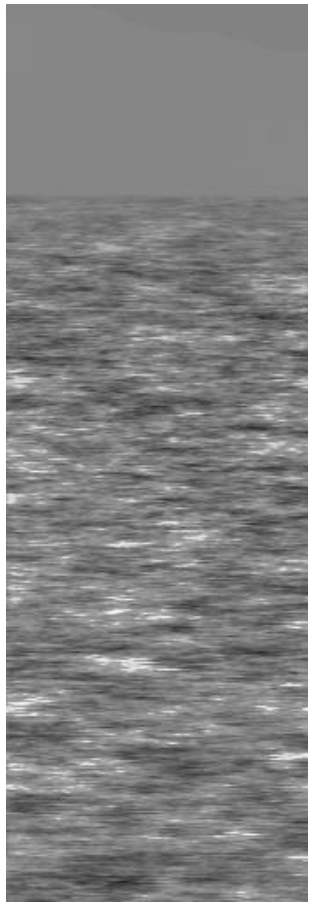

d)

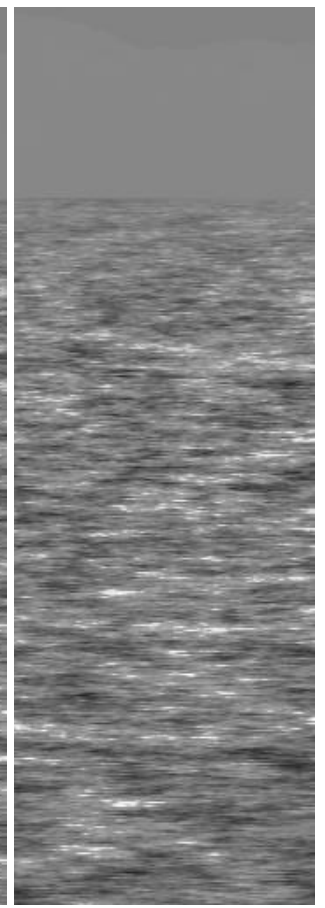

e)

Figure 6. MIBS simulations of $1^{\circ} \times 3^{\circ} \mathrm{MWIR}$ sea and coastal images in meteorological conditions specified in Table 1 (top: MWIR, bottom: LWIR, from left to right condition a-e). 


\section{VARIABILITY OF TARGET CONTRAST}

There are many factors that contribute towards the signature and ability to detect a small target, in the visible to infrared optical bands, at sea. During day time, the sun is the biggest source of energy to heat the target and cause target reflections. The movement of the target and the disturbance of the sea surface results in a detectable bow and stern wake. Heat sources internal or external to the small target (e.g. engines) can also lead to detection and classification. Furthermore, exposed persons on the deck can many times be responsible for the highest thermal contrast from the small target.

The wake contrast is a complex signature depending on many factors of which the most important ones are the sea state, target speed, propulsion type, hull shape and size, line-of-sight geometry and sun position. Examples of the wake signature are shown in Figure 8 together with a horizontal plot through the target and background. The wake contrast can in many cases be bigger than target itself, both in size and in intensity.
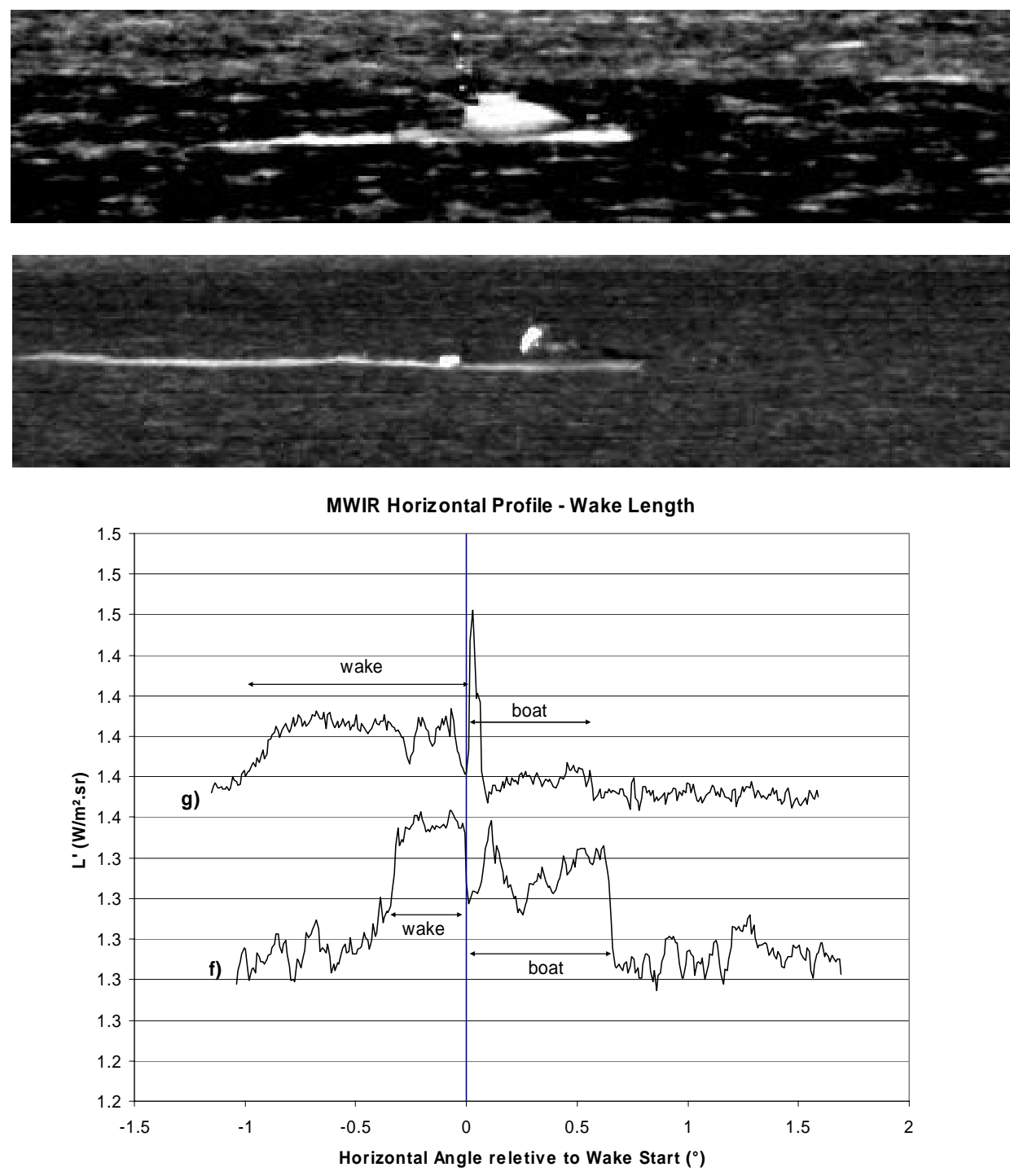

Figure 8. Examples of Wake Contrast in the MWIR (f upper image, g lower image).

Examples of other target features that can enhance detection are shown in Figure 9 and include sunlight reflections (from highly reflective surfaces and the hull itself) and exposed persons onboard. 

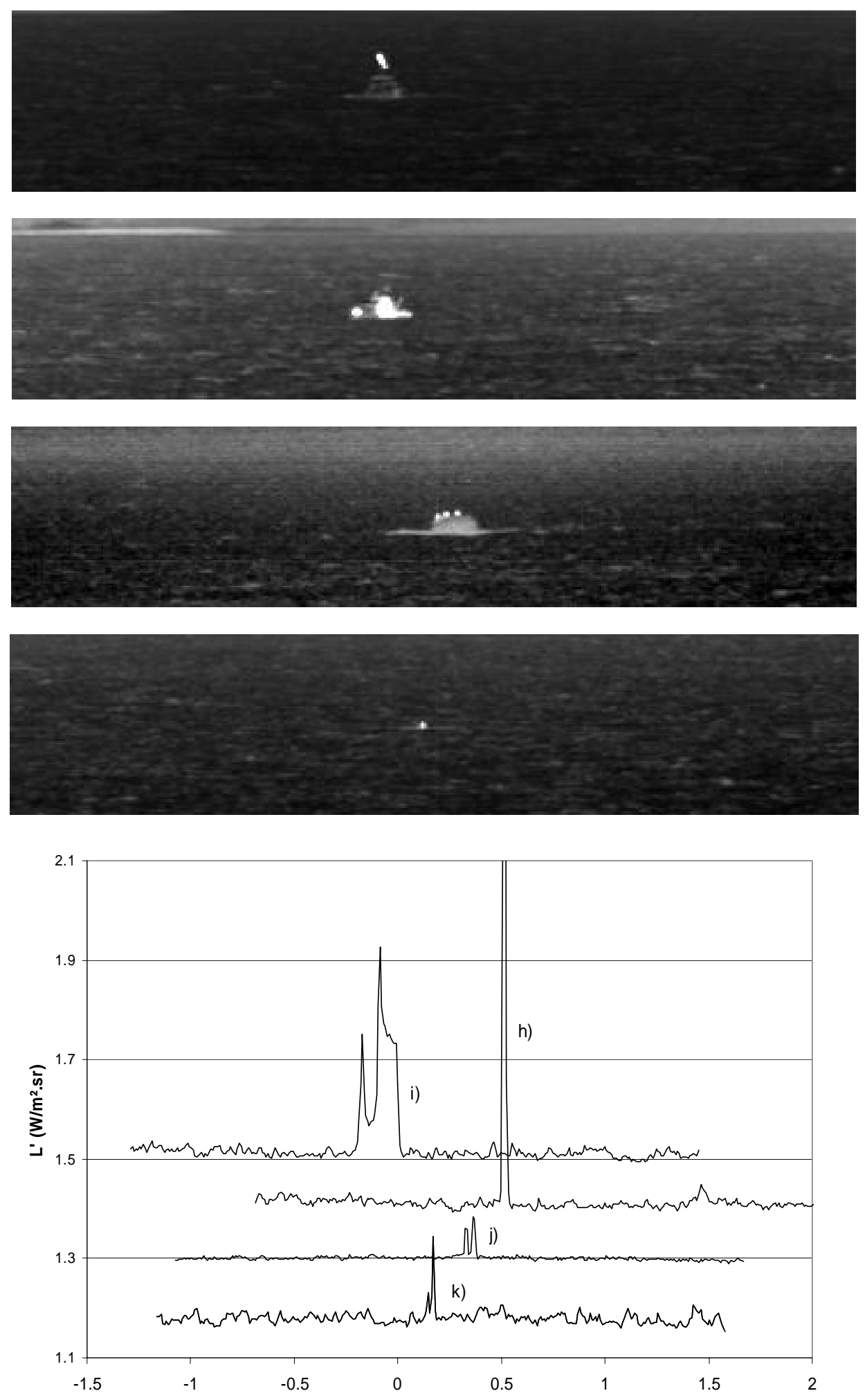

Figure 9. Target features that can enhance detection: from top to bottom h), i) reflections and, $j$ ), $k$ ) - exposed persons. 


\section{DERIVATION OF SENSOR MANAGEMENT INFORMATION}

We analyzed sea and land backgrounds extracted from imagery captured with the four imagers listed in Table 2 . These imagers cover the visual, short-, medium-, and long-wave infrared bands. The main feature of interest was the complexity of the backgrounds as presented in the four different optical bands. The analysis described here is based on Yang et $a l^{6}$. They described a criterion known as the variance weighted information entropy (WIE) to evaluate the complexity of sea-sky infrared (IR) backgrounds. The motivation for this criterion is modelled on the human's subjective perception that the more variance there is in the gray values in an image, the more complex the scene is, and therefore the more difficult it becomes to perform target tracking. The WIE criterion is summarized as follows:

Let $S$ denote the set of gray (digital) values present in an image, with $N$ discrete levels, and $p_{s}$, the probability of gray value $s$ occurring in the set $S$. The WIE of the image is then, $H(S)=-\sum_{s=0}^{N-1} s \cdot p_{s} \ln p_{s}$ with the condition that when $p_{s}=0$, then $p_{s} \ln p_{s}=0$. The variance WIE sets out to account for the variance of gray levels in the image and is given by $H(S)=-\sum_{s=0}^{N-1}(s-\bar{s})^{2} \cdot p_{s} \ln p_{s}$ again with the condition that when $p_{s}=0$, then $p_{s} \ln p_{s}=0$, and with $\bar{s}$ the mean of the gray values in the image. We selected sea and land backgrounds from representative imagery captured in the four spectral bands and compared the temporal behaviour of the variance WIE's. Whereas Yang et al investigated variations of the WIE criterion to determine which variation would perform the best in automatic small target detection schemes, our aim is to illustrate how the WIE criterion could aid in selecting the most appropriate sensor available, for automatic small target detection schemes. Figure 10 shows the scenario that was used to select a sea and land background for the analysis presented here. The weather during the experiment was cool with light rain and broken cloud. The broken cloud led to changing illumination in the scene.

Table 2: Imagers used during the trial for recording target and background optical characteristics

\begin{tabular}{|l|l|l|}
\hline Imager & Wavelength & Sensor Type \\
\hline Cedip Jade SWIR & $1.0-2.5 \mu \mathrm{m}$ & CMT detector; 14-bit \\
\hline Cedip Jade MWIR & $3.5-5.1 \mu \mathrm{m}$ & CMT detector; 14-bit \\
\hline Cedip Jade VLWIR & $7.8-12.0 \mu \mathrm{m}$ & CMT detector; 14-bit \\
\hline Prosilica & $612-652 \mathrm{~nm}$ & Silicon detector; 8-bit \\
\hline
\end{tabular}

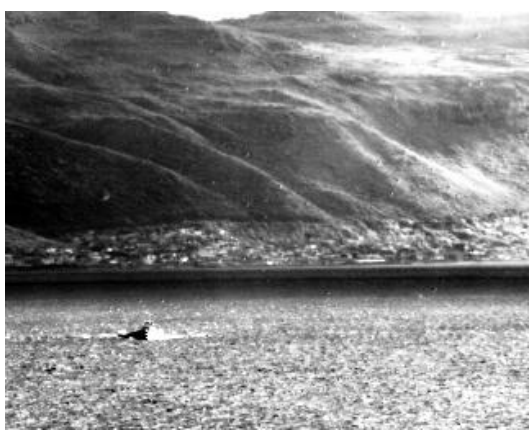

Figure 10. Short-wave infrared image of the scene used for WIE analysis

Figure 11 shows the temporal changes in the variance WIE for the different imagers. Observing the results for the visual and SWIR bands, similar trends are evident for the land and sea backgrounds, namely a reduction in the variance WIE for the sea background and an increase in the variance WIE for the land background. The variation is visible in the original imagery and is caused by changes in the illumination. In the scene presented here, the subjective conclusion is that the visual band is the most appropriate band for automatic target tracking. This type of information can be fed into system concepts making use of adaptive sensor management system such as described in Schwering et al. (2007) ${ }^{7}$. In particular temporal changes are relevant to adaptive systems. 

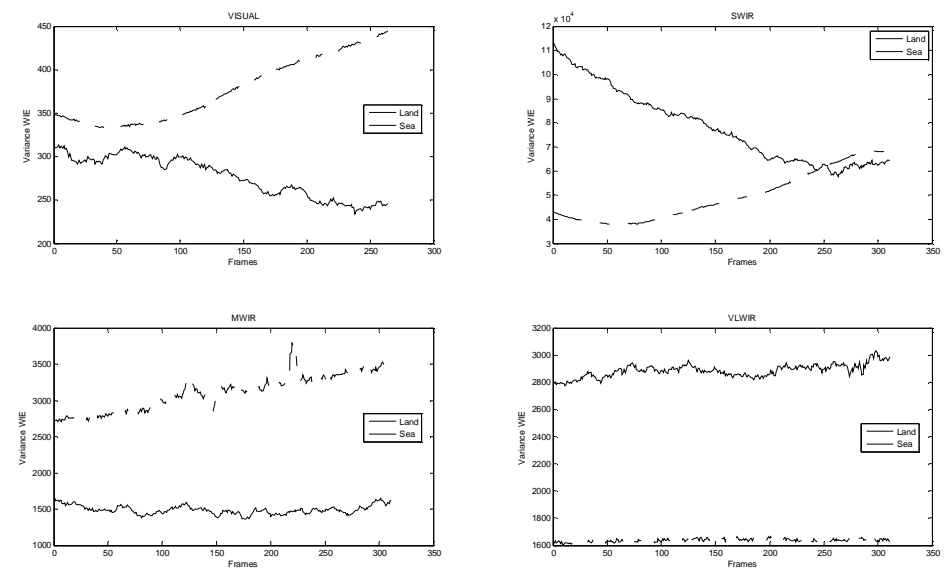

Figure 11. Results of temporal variance WIE analysis

\section{CONCLUSIONS}

We have presented our analysis of infrared backgrounds in bay environments. Data that was recorded at False Bay were analysed for variation in backgrounds, in vertical profile radiance and clutter. It was derived that the background is highly variable, and clutter levels can be extensive. Additional analysis of land, birds, sunglint gave more insight into these clutter phenomena. MIBS background simulations were performed for the scenario of these measurements conditions. The analysis is also given, and show accurate clutter predictions in coastal backgrounds. Specific variation in coastal radiance levels is underestimated by the simulation model. Furthermore an analysis was given into an approach to derive sensor management information to adapt a system towards most information content.

\section{ACKNOWLEDGEMENTS}

The work for this paper was supported by the Netherlands MoD under the programme V602 "Electro-Optical sensor systems" and in South Africa by Armscor. Data was recorded at IMT in False Bay (South Africa). The South African Navy assisted with the execution of the trial and their support is gratefully acknowledged. We acknowledge all authors of the third reference for recording and analysis of these data and for their contribution to the trials.

\section{REFERENCES}

1. Schwering, P.B.W., 2006, IRST evaluation methodologies: Maritime infrared background simulator, SPIE Proc. Vol. 6206-71, 620621, 2006

2. $\quad$ van den Broek, S.P., Bakker, E.J., de Lange, D.J., Theil, A; Detection and classification of infrared decoys and small targets in a sea background, 2000, SPIE Vol. 4029, 70-80, 2000

3. Schwering P.B.W., Bezuidenhout D.F., Gunter W.H., de Jong A.N., Fritz P.J., le Roux F.P.J., Sieberhagen R.H., Holloway M., Vrahimis G., October F.J., Kemp R.A.W., Optical Characterisation of small surface targets, SPIE Proc. 6739A-17, 67390H, 2007

4. Schwering, P.B.W., 1996, Maritime Infrared Background Clutter, SPIE Vol. 2742, pp255-266, 1996

5. Schwering, P.B.W., 1991, Infrared clutter measurements of marine backgrounds, SPIE Vol. 1486, pp25-36, 1991

6. Yang, L., Yang, J., Ling J., New criterion to evaluate the complex degree of sea-sky infrared backgrounds, Opt. Eng. 44(12) (2005), 126401-1 - 126401-5

7. Schwering, P.B.W., van den Broek, S.P., van Iersel, M., EO System Concepts in the Littoral, SPIE Proc. Vol. 6542-100, 654230, 2007 